\title{
Review
}

\section{Peptide toxins and small-molecule blockers of BK channels}

\author{
Mu YU ${ }^{1, \#}$, San-ling LIU ${ }^{1, \#}$, Pei-bei SUN², Hao PAN², Chang-lin TIAN, 2, *, Long-hua ZHANG, * \\ ${ }^{1}$ High Magnetic Field Laboratory, Chinese Academy of Sciences, Hefei 230031, China; ${ }^{2}$ School of Life Sciences, University of Science \\ and Technology of China, Hefei 230027, China
}

\begin{abstract}
Large conductance, $\mathrm{Ca}^{2+}$-activated potassium (BK) channels play important roles in the regulation of neuronal excitability and the control of smooth muscle contractions. BK channels can be activated by changes in both the membrane potential and intracellular $\mathrm{Ca}^{2+}$ concentrations. Here, we provide an overview of the structural and pharmacological properties of BK channel blockers. First, the properties of different venom peptide toxins from scorpions and snakes are described, with a focus on their characteristic structural motifs, including their disulfide bond formation pattern, the binding interface between the toxin and BK channel, and the functional consequence of the blockage of BK channels by these toxins. Then, some representative non-peptide blockers of BK channels are also described, including their molecular formula and pharmacological effects on BK channels. The detailed categorization and descriptions of these BK channel blockers will provide mechanistic insights into the blockade of BK channels. The structures of peptide toxins and non-peptide compounds could provide templates for the design of new channel blockers, and facilitate the optimization of lead compounds for further therapeutic applications in neurological disorders or cardiovascular diseases.
\end{abstract}

Keywords: BK channel; peptide toxins; scorpion venom; iberiotoxin; small-molecule blockers; alkaloids

Acta Pharmacologica Sinica (2016) 37: 56-66; doi: 10.1038/aps.2015.139

\section{Introduction}

BK channels are a group of calcium-activated potassium channels with large conductance that are found in both excitable and non-excitable cells. BK channels play important roles in many cellular processes, such as the regulation of neuronal firing and neurotransmitter release ${ }^{[1]}$, smooth muscle contractions $^{[2]}$, endocrine cell secretion ${ }^{[3,4]}$, and cell proliferation $^{[5-7]}$. BK channels are also called Maxi-K, slo1, KCNMA1, or $\mathrm{K}_{\mathrm{ca}} 1.1^{[8]}$. In general, a functional $\mathrm{BK}$ channel is formed by a tetramer of four pore-forming a subunits alone or in association with auxiliary $\beta$ subunits $(\beta 1-\beta 4)$ that play a modulatory role in specific tissues ${ }^{[9]}$. In contrast to other voltage-gated potassium channels, which have six transmembrane helices (S1-S6), the a subunit of BK channels possesses an extra transmembrane domain (S0 at the N-terminus) and a unique long, intracellular tail at the C-terminus (Figure 1). The S0 segment assembles with the $\beta$ subunit and may contribute to the regu-

\footnotetext{
\#These authors contributed equally to this work.

* To whom correspondence should be addressed.

E-mail zlhustc@ustc.edu.cn (Long-hua ZHANG); cltian@ustc.edu.cn (Chang-lin TIAN)

Received 2015-09-15 Accepted 2015-10-13
}

lation of the functions of the voltage-sensing domain ${ }^{[10-12]}$. The long intracellular tail at the C-terminus forms a gating ring that can be triggered by intracellular chemical ligands, including $\mathrm{Ca}^{2+}, \mathrm{Mg}^{2+}$, and lipid molecules ${ }^{[13-15]}$. BK channels act as molecular transducers that link intracellular calcium signals with extracellular stimuli, and an allosteric link between the opening of the voltage-activated channel and calcium sensitivity has been proposed ${ }^{[16,17]}$.

Laboratory and clinical studies have suggested that the malfunction of BK channels may cause numerous disorders in humans, such as hypertension ${ }^{[18]}$, asthma ${ }^{[19]}$, diabetes ${ }^{[20]}$, epilepsy ${ }^{[21]}$ and cancer ${ }^{[22]}$. Either "gain-of-function" or "loss-offunction" mutations are identified in both the $\alpha$ and $\beta 1$ subunits of BK channels. The $\beta 1$ subunit appears to play a major role in regulating the sensitivity of BK channels intracellular calcium spikes. Impairment of the $\beta 1$ subunit of BK channels results in an increase in arterial tone and blood pressure or hydrosaline retention in the kidney. These unique characteristics of BK channels represent specific targets for drug development.

A number of peptide toxins targeting BK channels have recently been isolated from various venoms, particularly scorpion venom. Because of their modest size, compact structure, and high potency and selectivity, these venom toxins are widely used as pharmacological probes to determine the 


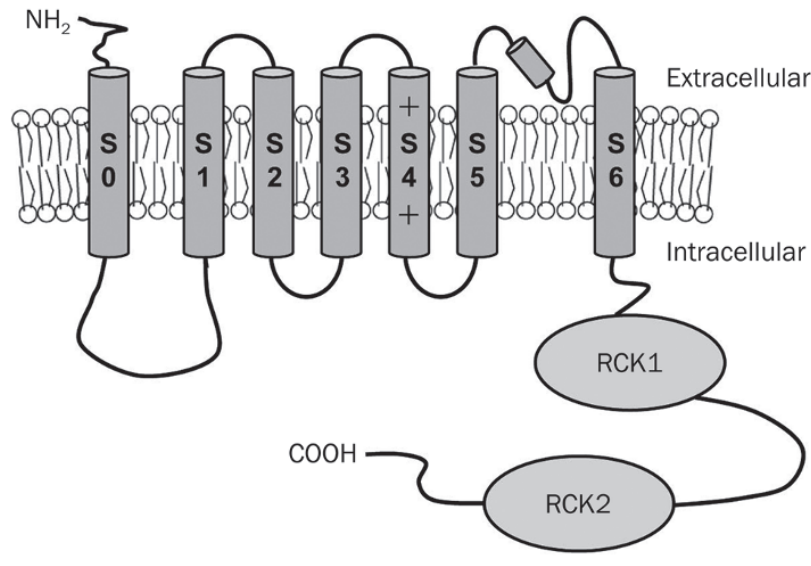

Figure 1. Topology of the BK channel $\alpha$ subunit (KCNMA1). So-S6 corresponds to the seven transmembrane segments of the $\alpha$ subunit, where SO is thought to be important in facilitating the association of the $\alpha$ and $\beta$ subunits. RCK1 and RCK2 in the cytosolic domains represent binding sites for $\mathrm{Mg}^{2+}$ and $\mathrm{Ca}^{2+}$, respectively.

molecular structure of BK channels and design therapeutic agents.

\section{Peptide toxin blockers for BK channels}

Among the different types of peptide toxins, the a-KTx peptides block potassium channels with high affinity and specificity. These peptides can be divided into at least 19 subfamilies based on their amino acid sequences, and several members of these subfamilies have a high affinity for BK, SK, and IK channels. Theses peptide toxins are pore blockers that are used to identify the pore domain of BK channels. BK blockers are used to probe BK channel function and therapeutic utility. The following section presents an overview of the peptide toxins and describes their different channel affinities and threedimensional structures.

\section{$\mathrm{CS} \alpha / \beta$ toxins in scorpions}

Scorpion venoms are dominated by so-called "cysteinestabilized $\alpha / \beta(\mathrm{CS} \alpha / \beta)$ " toxins that are evolutionarily derived from $\mathrm{CSa} / \beta$ defensins. Most peptide toxins that target BK channels are from scorpions. These toxins are pore-blocking toxins that inhibit the flow of $\mathrm{K}^{+}$ions through BK channels by plugging the ion conductance pathway with a highly conserved lysine residue. Based on the length of the amino acid sequence and the number of cysteine residues, these toxins can be classified into several types: short-chain peptide toxins of 29-39 amino acid residues with three disulfide bridges, including a-KTx1, a-KTx3, a-KTx16, and a-KTx19; short-chain peptides with eight disulfide bridges, including a-KTx12; and long-chain peptide toxins containing 60-70 amino acid residues with four disulfide bridges, including the recently discovered BmP09 peptide toxin.

\section{The $\alpha-K T x$ 1.x subfamily}

The a-KTx 1.x subfamily is a group of short peptides consist-

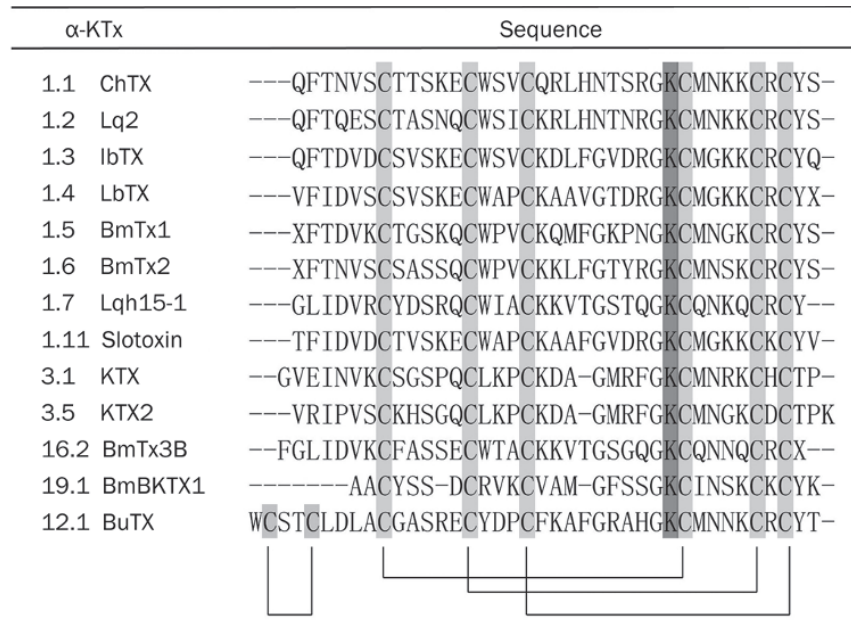

Figure 2. Multiple sequence alignment of short-chain peptide toxins from scorpion venom. Conserved cysteine and lysine residues are designated by a dark background. The disulfide framework and the extra disulfide bridge in BuTx are labeled, along with their sequences.

ing of 36-37 amino acid residues and three disulfide bridges. This subfamily displays high affinity for BK channels ${ }^{[23]}$. The " $x$ " refers to the chronological order in which the peptide was discovered. Within subfamily 1 , only a-KTx 1.3 (iberiotoxin, $\mathrm{IbTX})^{[24,25]}$ and a-KTx 1.4 (limbatustoxin, LbTX) ${ }^{[26,27]}$ have high selectivity for BK channels, whereas a-KTx 1.1 (charybdotoxin, ChTX) $)^{[28]}$ and a-KTx $1.2\left(\right.$ Lq2 ${ }^{[29,30]}$ block BK channels and other types of voltage-gated potassium channels. Although the selectivity and affinity of these toxins differ, they exhibit a high degree of sequence similarity (Figure 2). Each of these toxins have a $\mathrm{W}$ residue at position 14 and a $\mathrm{K}$ residue at position 27 and contain six conserved cysteine residues in three disulfide bridges with $\mathrm{C}^{\mathrm{I}}-\mathrm{C}^{\mathrm{IV}}, \mathrm{C}^{\mathrm{II}}-\mathrm{C}^{\mathrm{V}}$, and $\mathrm{C}^{\mathrm{III}}-\mathrm{C}^{\mathrm{VI}}$ connectivity to maintain the rigid structure ${ }^{[28,31]}$. Their structures share a classic motif containing a $\beta$-sheet formed by three anti-parallel $\beta$-strands on one side of the molecule and a helix on the other side $^{[32]}$ (Table 1).

The binding of toxins to BK channels is very sensitive to electrostatic interactions between several basic residues on the toxin and negative charges in the outer vestibule of the channel ${ }^{[33,34]}$. Multiple residues, including Trp14 on the $\beta$-sheet face of the helix ${ }^{[35]}$, Thr23 and Arg25 in the turn between the helix and the second anti-parallel strand ${ }^{[36]}$, and Lys27, Arg34, and Tyr36 in the second and third strands of the $\beta$-sheet ${ }^{[35-37]}$, are critical for binding of toxins to the BK channel. This region is highly conserved within the a-KTx $1 . x$ subfamily. Therefore, the primary sequence and the three-dimensional structure of the toxins are important determinants for recognizing and interacting with BK channels.

Charybdotoxin, also called ChTX, was the first potent peptide toxin used to identify and characterize a BK channel ${ }^{[23]}$. ChTX is a 37-amino-acid peptide from the venom of the scorpion Leiurus quinquestriatus (var. hebraeus). ChTX has eight positively charged residues, two negatively charged residues, and a blocked N-terminus (cyclization of glutamine at the 


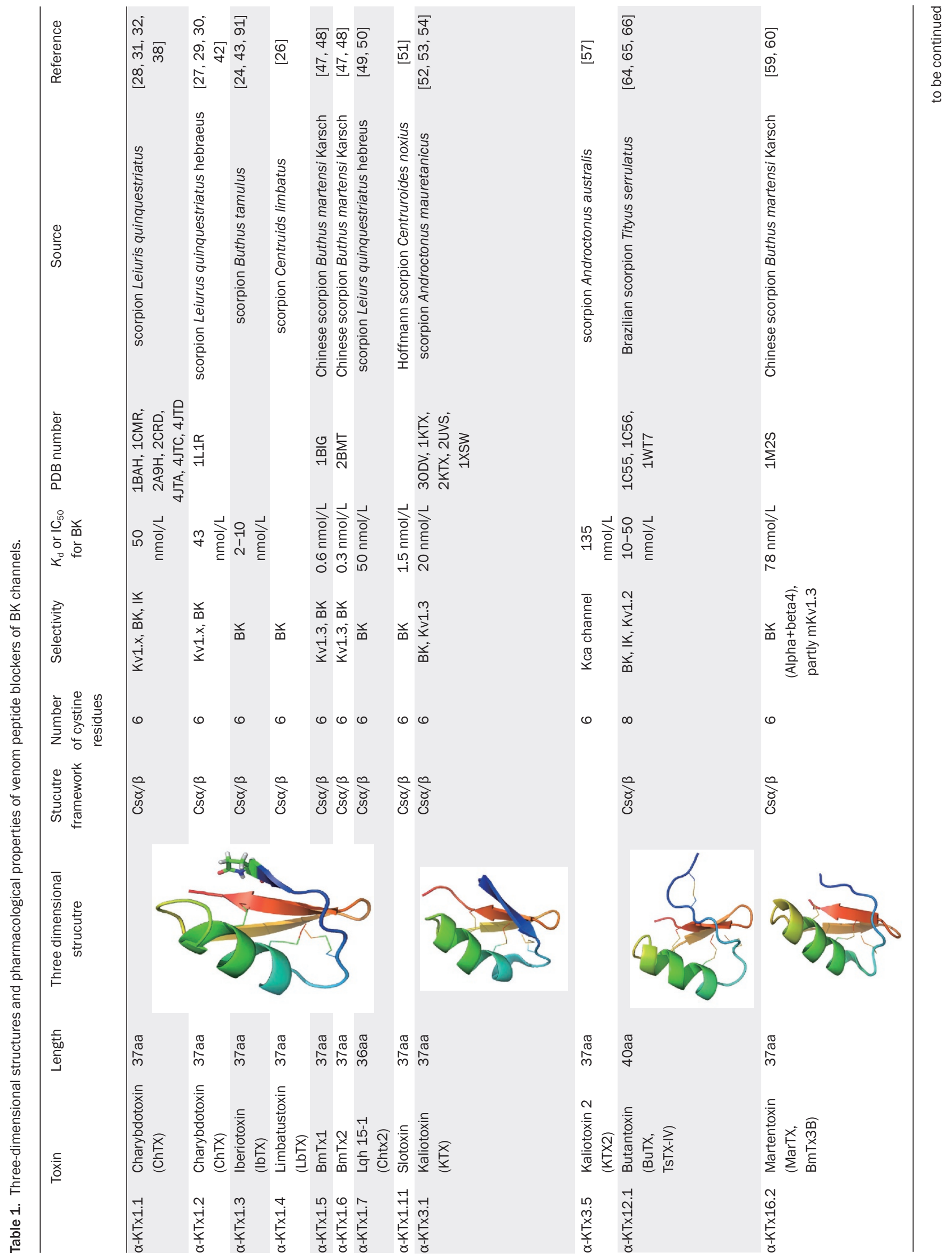




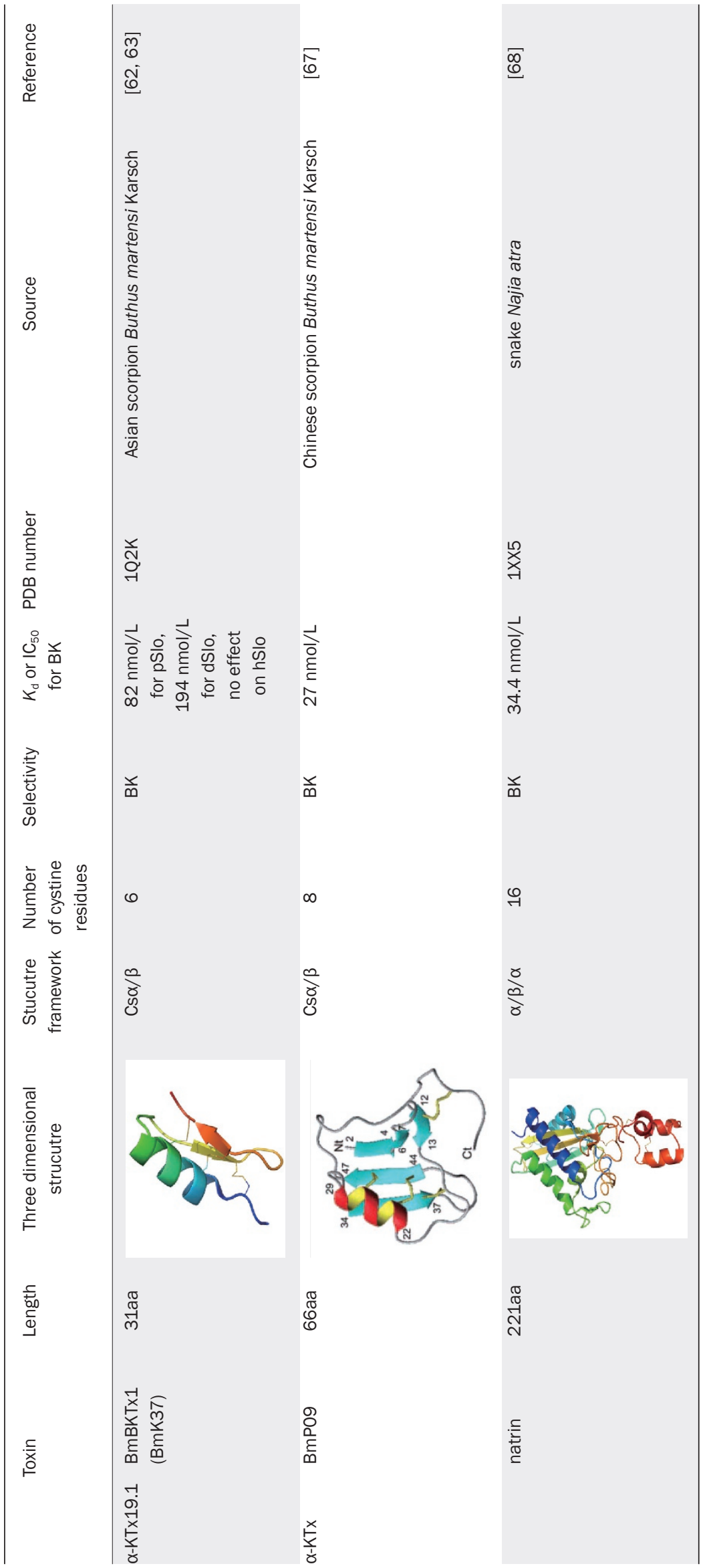


N-terminus). ChTX can bind to the outer vestibule of a BK channel, but this binding is greatly decreased by an increased internal potassium concentration. Unfortunately, ChTX also blocks other subtypes of potassium channels, such as the smallconductance, calcium-activated potassium channel $(\mathrm{SK})^{[38]}$, HLK3 (a voltage-gated potassium channel) ${ }^{[39]}$, and a modified Drosophila "Shaker" $\mathrm{K}^{+}$channel $^{[40]}$. The crystal structure of this peptide toxin in complex with a $\mathrm{Kv}$ channel indicates that ChTX binds to the channel in a lock and key manner and interacts directly with conducting ions inside the selectivity filter ${ }^{[41]}$.

Lq2 (a-KTx 1.2) is a ChTX homolog derived from the venom of the Israeli scorpion, Leiurus quinquestriatus hebraeus. In contrast to ChTX, Lq2 also contains 37 amino acid residues and a classic $\alpha / \beta$ scaffold $^{[30]}$. Lq2 blocks BK channels with a $K_{D}$ of 43 $\mathrm{nmol} / \mathrm{L}^{[29]}$ and can also block inwardly rectifying potassium channels ${ }^{[42]}$.

IbTX (a-KTx 1.3, iberiotoxin), a minor fraction of the crude venom of Buthus tamulus, was discovered by Galvez et al in $1990^{[24]}$. IbTX also consists of 37 amino acids and possesses $68 \%$ sequence identity with $\mathrm{ChTX}^{[24,25]}$. IbTX has one less positively charged and four more negatively charged residues than ChTX. Functional studies have demonstrated that IbTX binds to the external opening of BK channels with higher affinity than ChTX as indicated by the lower dissociation rate of IbTX compared with $\mathrm{ChTX}^{[24,25]}$. The nuclear magnetic resonance (NMR) structure of synthetic IbTX suggests that the configuration of the peptide backbone is nearly identical to that of ChTX. IbTX is a specific blocker of BK channels and is widely used in structural and functional studies of BK channels ${ }^{[24,43-46]}$.

LbTX (a-KTx 1.4, limbatustoxin), was isolated from the scorpion Centruroides limbatus. LbTX shares 57\% and 70\% sequence identity with ChTX and IbTX, respectively. LbTX also exhibits high selectivity and affinity for BK channels ${ }^{[26]}$.

BmTx1 (a-KTx 1.5) and BmTx2 (a-KTx 1.6) are both 37 -amino-acid peptide toxins purified from the venom of the Chinese Buthid (Buthus martensii). The solution structures of these two peptides are very similar ${ }^{[47]}$. They both contain a triple-stranded $\beta$-sheet connected to an a-helix, a classic motif in a-KTx 1 subfamily structures. The inhibitory effects of BmTx1 and BmTx2 on BK channels are similar, with $\mathrm{IC}_{50}$ values of $0.65 \mathrm{nmol} / \mathrm{L}$ and $0.3 \mathrm{nmol} / \mathrm{L}$, respectively ${ }^{[48]}$. These toxins also block voltage-gated potassium channels.

Lqh 15-1 (a-KTx 1.7), also called ChTx2, was isolated from the scorpion venom of Leiurus quinquestriatus hebraeus ${ }^{[49,50]}$. Lqh 15-1 consists of 36 amino acid residues. ChTX (a-KTx 1.1) and Lqh 15-1 are homologues with similar three-dimensional structures. Depending on the conditions, Lqh 15-1 blocks BK channels with $K_{\mathrm{D}}$ values of $50 \mathrm{nmol} / \mathrm{L}^{[50]}$ or $105 \mathrm{nmol} / \mathrm{L}^{[49]}$.

Slotoxin ( $a-K T x$ 1.11), which contains 37 amino acids, was isolated from Centruroides noxius Hoffmann scorpion venom. Slotoxin specifically blocks mammalian BK channels (hslo) ${ }^{[51]}$. Slotoxin also can differentiate among the three types of BK complexes, including $\alpha, \alpha+\beta 1$, and $\alpha+\beta 4$. For example, slotoxin reversibly blocks the pore-forming a subunit with a $K_{D}$ of $1.5 \mathrm{nmol} / \mathrm{L}$, but the blocking effects on the $\alpha+\beta 4$ complexes are much weaker, with a $K_{\mathrm{D}}$ of $100 \mathrm{nmol} / \mathrm{L}$.

\section{Kaliotoxin ( $\alpha-K T x$ 3.x)}

Kaliotoxin(a-KTx 3.1) is a neurotoxin purified from the scorpion Androctonus mauretanicus mauretanicus ${ }^{[52]}$. Kaliotoxin (KTX) has 37 amino acid residues and shares 38\% identity with ChTX and $44 \%$ identity with IbTX. Because of the presence of two prolines in the a-helix, the three-dimensional structure of KTX determined by NMR includes a shorter and distorted a-helix that differs slightly from those of its homologues ${ }^{[53]}$. Furthermore, the increased length of the extended structure preceding the helix alters the accessibility of the conserved Lys27 $7^{[54]}$ and, consequently, its channel blocking effect ${ }^{[55]}$. KTX can block both Kv1.3 ${ }^{[56]}$ and BK channels ${ }^{[52]}$ with a $K_{\mathrm{D}}$ of 20 $\mathrm{nmol} / \mathrm{L}^{[52]}$.

Kaliotoxin 2 (KTX2, a-KTx 3.5) also contains 37 amino acid residues and shares $76 \%$ identity with KTX. These two peptides differ mainly in their N-terminal regions and residues, such as 31 and 34, that are located in the channel recognition region. These differences result in a 5-fold decrease in channel blocking by KTX2 compared with $\mathrm{KTX}^{[57]}$.

\section{Martentoxin ( $\alpha-K T x$ 16.2)}

Martentoxin (a-KTx 16.2), also named MarTX or BmTx3B, is a potassium channel blocker derived from the Chinese scorpion Buthus martensii Karsch. Martentoxin has 37 amino acid residues and forms a typical $\mathrm{CSa} \beta$ motif with three disulfide bridges involving Cys8-Cys29, Cys14-Cys34, and Cys18Cys36. Although the structure of martentoxin is similar to that of the a-KTx1 subfamily, the electrostatic and hydrophobic surface distributions differ considerably ${ }^{[58]}$. Martentoxin blocks the currents of neuronal BK channels with an $\mathrm{IC}_{50}$ of $78 \mathrm{nmol} / \mathrm{L}$ in $\mathrm{Ca}^{2+}$-dependent manner and partially inhibits mKv1.3 channels ${ }^{[59-61]}$.

\section{BmBKTX1 ( $\alpha-K T x$ 19.1)}

BmBKTX1 (a-KTx 19.1) is a 31-amino-acid peptide derived from the Chinese scorpion Buthus martensii Karsch. Twodimensional NMR spectroscopy revealed a typical Csa $\beta$ motif for BmBKTX1 ${ }^{[62]}$. However, BmBKTX1 has only two $\beta$-sheet strands connected to an a-helix with three disulfide bridges involving Cys3-Cys22, Cys8-Cys27, and Cys12-Cys29. BmBKTX1 blocks the a subunits of the BK channels of the cockroach (pSlo) and fruit fly (dSlo) with $\mathrm{IC}_{50}$ values of $82 \mathrm{nmol} / \mathrm{L}$ and $194 \mathrm{nmol} / \mathrm{L}$, respectively. However, BmBKTX1 does not affect the activity of human BK channels (hSlo) or other voltage-gated potassium channels ${ }^{[63]}$.

\section{Butantoxin ( $\alpha-K T x$ 12.1)}

Butantoxin (a-KTx 12.1), also named BuTX or TsTX-IV, is present in the venom of three Brazilian scorpions, Tityus serrulatus, Tityus trivittatus, and Tityus stigmurus. Butantoxin consists of 40 amino acid residues with four disulfide bridges: Cys2-Cys5, Cys10-Cys31, Cys16-Cys36, and Cys20-Cys38. The secondary structure of butantoxin contains a short, two-turn a-helix (Glu15-Phe23) and a two-stranded $\beta$-sheet (Gly29-Met32 and Lys35-Cys38) ${ }^{[64]}$. Butantoxin contains more disulfide bonds than other $\mathrm{CS} \alpha / \beta$ toxins. The disulfide bonds $\mathrm{C}^{\mathrm{III}}-\mathrm{C}^{\mathrm{VI}}, \mathrm{C}^{\mathrm{IV}}-\mathrm{C}^{\mathrm{VII}}$, 
and $\mathrm{C}^{\mathrm{V}}-\mathrm{C}^{\mathrm{VIII}}$ constitute the core disulfide framework of $\mathrm{CSa} / \beta$ defensins. The remaining $N$-terminal $C^{\mathrm{I}}-\mathrm{C}^{\mathrm{II}}$ disulfide bridge is unique to this toxin and does not appear to confer additional stability $^{[64]}$. Butantoxin blocks Kv1.2 ${ }^{[65]}$ and BK channels ${ }^{[66]}$ with $\mathrm{IC}_{50}$ values of $165 \mathrm{nmol} / \mathrm{L}$ and $50 \mathrm{nmol} / \mathrm{L}$, respectively.

\section{The BmP09 long-chain toxin}

BmP09, purified from the venom of the Chinese scorpion Buthus martensii Karsch, is a novel long-chain toxin. BmP09 contains 66 amino acid residues, including eight cysteines that specifically block the subunits of mSlo1 with an $\mathrm{IC}_{50}$ of $27 \mathrm{nmol} / \mathrm{L}$. BmP09 exhibits greater specificity and reversibility than ChTX. The Met66 residue at the C-terminus is very important for maintaining selectivity for BK channels. A three-dimensional simulation suggests that the interaction between BmP09 and the BK channel is stabilized by aromatic $\Pi-\Pi$ interactions. The Lys41 residue of BmP09 also blocks the pore of the entrance of the BK channel ${ }^{[67]}$.

\section{$\alpha / \beta / \alpha$ toxins from snakes}

Although scorpion venoms are a rich source of peptide toxins that interact with BK channels, many potent and selective BK blockers have been extracted from other species, such as natrin from Naja atra venom ${ }^{[68]}$. Natrin is a member of the cysteinerich secretory protein (CRISP) family that is found in many snake venoms ${ }^{[69]}$. It consists of 221 amino acid residues that form two separate domains, the N-terminal $\alpha / \beta / \alpha$-sandwich motif (PR-1 domain, residues 1-160) and the C-terminal cysteine-rich domain (CRD, residues 183-221). A compact hinge region (residues 161-182) connects these two domains. All members of the CRISP family contain 16 strictly conserved cysteines that form eight disulfide bonds. Some members of the CRISP family block L-type $\mathrm{Ca}^{2+}$ channels or cyclic nucleotide-gated ion channels. However, natrin can block BK channels in a concentration-dependent manner with an $\mathrm{IC}_{50}$ of 34.4 $\mathrm{nmol} / \mathrm{L}^{[68]}$. The flexible CRD domain is thought to play an important role in channel blocking.

\section{Use of peptide toxins for structural and functional analyses}

Native toxins from animal venoms are usually present in small amounts, but chemical synthesis or recombinant expression in bacteria can provide large amounts of the toxin ${ }^{[0,71]}$. The expression of fusion-tagged toxins using a bacterial system is the most economical method of producing these toxins, but cleavage of the fusion tags normally results in extra residue(s) at the N-terminus ${ }^{[72]}$. The extra residues are thought to have little or no influence on toxin activity. We describe the preparation of IbTX as an example of a recombinant expressed toxin to illustrate the influence of extra residues at the N-terminus.

IbTX is a typical peptide toxin with high selectivity and affinity for BK channels. To obtain large amounts of IbTX, two SUMO-tagged IbTXs with either a SUMO protease or a Factor Xa cleavage site at the N-terminus of the IbTX peptide were cloned, expressed, and purified. Cleavage by SUMO protease introduced an extra serine encoded by the internal part of the BamHI restriction site. Thus, the N-terminal sequence of this SIbTX begins with SQ. Factor Xa cleavage produced an IbTX with an N-terminal $Q$ that was cyclized to pyroglutamate (cIbTX). The toxins and their three disulfide bonds were verified by HPLC and ESI-MS. Patch-clamp electrophysiological assays of SIbTX or cIbTX against a BK channel expressed in Chinese hamster ovary cells demonstrated that $1 \mu \mathrm{mol} / \mathrm{L}$ cyclized IbTX (cIbTX) reduced the BK current to $17 \%$ of the control, with an $\mathrm{IC}_{50}$ of $14.9 \pm 1.4 \mathrm{nmol} / \mathrm{L}(n=5)$, whereas 1 $\mu \mathrm{mol} / \mathrm{L}$ uncyclized IbTX (sIbTX) only reduced the BK current to $40 \%$ of the control with an $\mathrm{IC}_{50}$ of $105.8 \pm 1.9 \mathrm{nmol} / \mathrm{L}(n=5)$ at a holding potential of $120 \mathrm{mV}$ (Figure 3). These results demonstrate that the affinity of cIbTX for BK channels is 10-fold higher than that of SIbTX, indicating that the presence of an extra residue at the N-terminus of IbTX strongly influences the self-cyclization, channel binding, and inhibitory activities of the toxin for the BK channel.

These results are consistent with the observation that the generation of pyroglutamic acid at the $\mathrm{N}$-terminus can greatly enhance the blocking effect of toxins for their target channels.



$1 \mu \mathrm{mol} / \mathrm{L} \mathrm{SIbTX}$

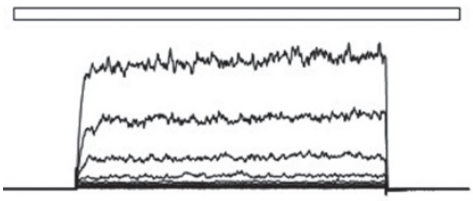

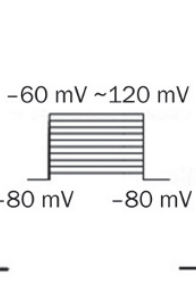

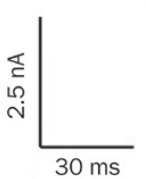

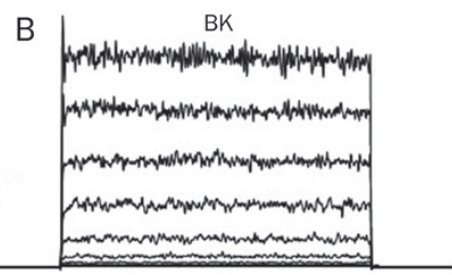

$1 \mu \mathrm{mol} / \mathrm{L} \mathrm{clbTx}$
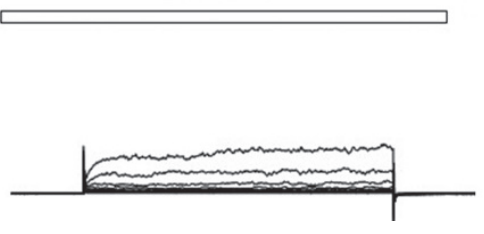

C

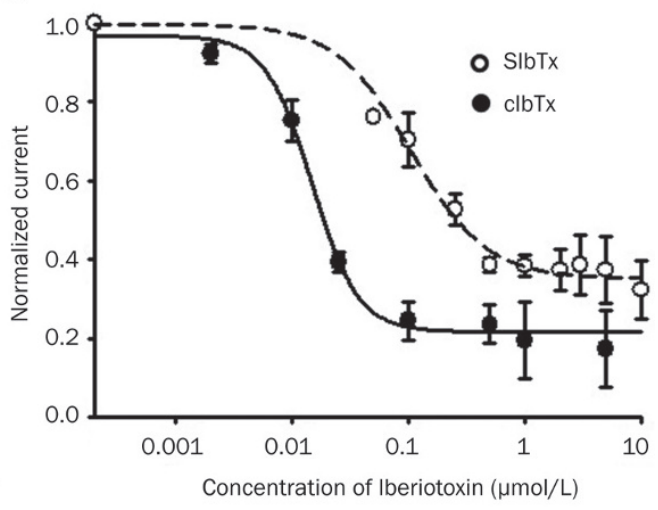

Figure 3. Blocking activity of SIbTx and clbTx on the BK channel. SIbTx denotes an extra S at the N-terminal region of IbTx, and clbTx denotes cyclization of the $\mathrm{N}$-terminal glutamine to pyroglutamate. 
This modification is present in many types of peptide toxins from diverse origins. Most of these modifications occur in conotoxin, whereas only a few occur in spider and scorpion toxins. Therefore, the $\mathrm{N}$-terminal generation of pyroglutamic acid is a major posttranslational modification of secretory proteins and peptides. This modification is important for biological function, such as mediating interactions with receptors or stabilizing against $\mathrm{N}$-terminal degradation ${ }^{[73]}$. Our results provide evidence that the $\mathrm{N}$-terminal generation of pyroglutamic acid by IbTX increases its ability to block BK channels.

\section{Non-peptide blockers}

Small-molecule blockers are another type of BK channel blockers. They are usually produced by various organisms, such as bacteria, fungi, plants, and animals, using various biosynthetic methods. In general, the vast majority of non-peptide blockers of BK channels are alkaloids, including indole diterpenes (paxilline, verruculogen, and penitrem), organic amines (tetraethylammonium, verapamil, and ketamine), quinoline derivatives (tetrandrine, quinine, and quinidine), and imidazole derivatives (clotrimazole). Although these small molecules are alkaloids, their structures are very diverse (Figure 4) and their interactions with ion channels vary dramatically ${ }^{[74-76]}$. The most potent and selective non-peptide blockers are tremorgenic indole-diterpenes from fungi of the genera Penicillium, Aspergillus, and Claviceps ${ }^{[77]}$.

\section{Indole diterpenes}

Tremorgenic indole diterpenes belong to a family of tremorgenic mycotoxins from fungi. They can cause neural disorders, such as staggers syndromes in ruminants ${ }^{[78,79]}$. These molecules are allosteric modulators, as determined by a $\left[{ }^{125} \mathrm{I}\right]$ ChTX binding assay, and may act in either a positive or negative manner ${ }^{[74]}$. One group inhibits the $\left.{ }^{[25} \mathrm{I}\right]-C h T X$ binding interaction, acting as a negative allosteric modulator, and includes paspalitrem A and C, aflatrem, penitrem A, and paspalinine. The other indole diterpenes are positive allosteric modulators that exhibit increased $\left[{ }^{125} \mathrm{I}\right]$-ChTX binding in a concentration-dependent manner, such as paxilline, verruculogen, and paspalicine. Furthermore, the $\left[{ }^{125} \mathrm{I}\right]-\mathrm{ChTX}$ binding assay revealed a unique interaction of these compounds through the $\beta$ subunit of the BK channel.

\section{Tetraethylammonium}

Organic amines, such as tetraethylammonium (TEA) and its analogues, were originally used to investigate the structure of the ion conduction pathway of BK channels expressed in the rat muscle membrane ${ }^{[80]}$. TEA and its analogues block BK channels in a voltage-dependent manner. However, as a widely used open-channel blocker of different types of voltage-gated potassium channels, the applicability of TEA for BK channel studies is limited. Furthermore, TEA and its analogues block BK channels through either the internal or external side of the planar lipid membrane, suggesting a different interaction mode compared with peptide blockers of the BK channel.

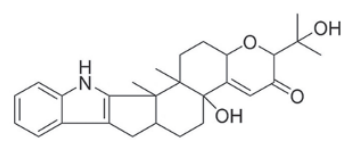

Paxilline

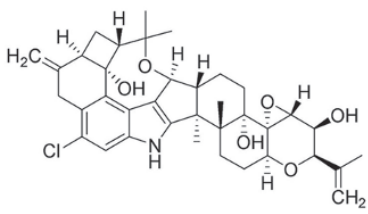

Penitrem A

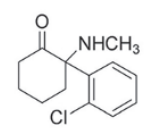

Ketamine
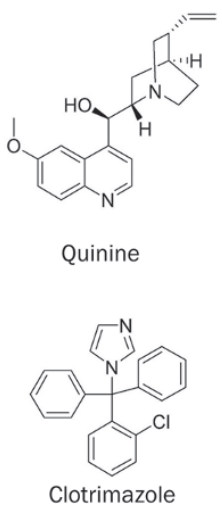

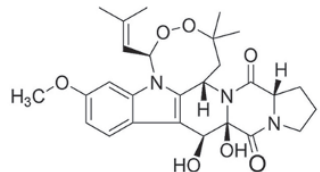

Verruculogen

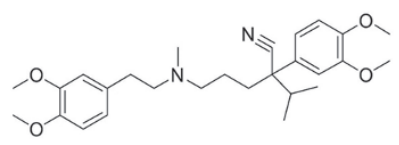

Verapamil
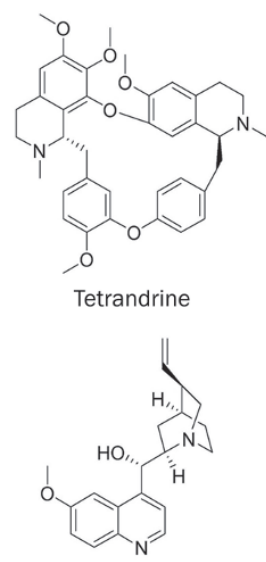

Quinidine

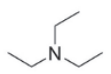

TEA
Figure 4. Molecular formulas of multiple non-peptide blockers of BK channels. Although these small-molecule blockers are alkaloids, with the exception of TEA, their structures vary dramatically.

\section{Other non-peptide blockers}

Alkaloids such as organic amines (verapamil and ketamine), quinoline derivatives (tetrandrine, quinine, and quinidine), and imidazole derivatives (clotrimazole) also possess BK channel blocking activity. Verapamil and its analogues gallopamil and D890, a type of calcium channel blocker, can induce a reversible flickering block of the BK channel activity in rat aortic myocytes ${ }^{[81]}$. Ketamine, a dissociative anesthetic, inhibits BK channels by an indirect mechanism. Quinine and quinidine, extracted from Cinchona species, can block both BK and IK channels ${ }^{[82,83]}$. Tetrandrine from the tuberous roots of Stephania tetrandra is a bis-benzylisoquinoline alkaloid that inhibits the activity of BK channels in isolated nerve terminals of rat neurohypophysis and cultured endothelial cells ${ }^{[84,85]}$. The antifungal clotrimazole, a member of the cytochrome P450 inhibitor family, can block BK activity without altering singlechannel conductance ${ }^{[86]}$.

Additional endogenous and exogenous compounds have 
been reported to inhibit BK channel activity. For example, 2-methoxyestradiol (an endogenous metabolite of 17$\beta$-estradiol) and ICI-182,780 (an intracellular estrogen receptor antagonist) can reduce channel activity by shifting the activation curve of the BK channel to a positive potential ${ }^{[87,88]}$.

Until recently, the pharmacology of the modulation of maxi$\mathrm{K}$ channels was restricted to potent blockade by the $\mathrm{K}^{+}$channel blocker TEA, effective and specific blockade by several scorpion toxin peptides, such as IbTX, and blockade by a series of tremorgenic alkaloids exemplified by paxilline ${ }^{[89]}$. Although none of these compounds have true potential as therapeutic agents, they continue to be very useful tools in the study of BK channels.

\section{Summary and perspective}

We have provided an overview of BK channel blockers, including the structures and pharmacological properties of venom peptides and the molecular formulas and pharmacological effects of non-peptide compounds.

\section{The structural properties and blocking effects of peptide blockers for BK channels}

In various scorpion venom peptide blockers of the BK channel, precise disulfide frameworks maintain the CSa/ $\beta$ motif, allowing the conserved lysine to block the entrance to the channel pore. The existence of an interaction surface, such as that of ChTX (a-KTx 1.1) ${ }^{[28,31,90]}$ and IbTX (a-KTx 1.3) ${ }^{[91]}$, assists binding to the extracellular opening of the BK channel conduction pathway and occludes the outer part of the channel pore, thereby optimizing the interaction between these venom peptides and their molecular targets. In addition to the typical a-KTx peptides that block potassium channels, snake venom peptides with different three-dimensional structures and disulfide bridge patterns also exhibit BK channel blocking properties. The CRD region of these peptides is thought to act as the channel blocker.

\section{Structural properties and BK channel binding affinity and specificity of peptide blockers}

The binding of a-KTx peptides to the BK channel is very sensitive to the electrostatic and hydrophobic surface distributions of the venom peptides. Sequence alignments of a-KTx peptides suggest a less positive net charge for IbTX, with four more negatively charged (Asp) and one less positively charged residue. In addition to the difference in electrostatic structure between ChTX and IbTX, Gly30 in IbTX is another determinant of its specificity for the $\mathrm{BK}$ channel ${ }^{[92,93]}$; this residue is also found in other BK channel-specific blockers, such as LbTX and Slotoxin. Furthermore, our studies of recombinant peptide toxins produced in bacteria have indicated that the pyroglutamic acid of the N-terminus of venom toxins may greatly enhance the blocking effects of toxins on target channels.

Thus, the surface charge distributions and three-dimensional structures of toxins are important determinants of their recognition and interactions with BK channels. This knowledge will facilitate the development of new toxin functions using similar three-dimensional scaffold and electrostatic surface distributions.

\section{Potential clinical applications for BK channel peptide blockers}

BK channels are important integrators in many biological systems, and their (patho) physiological functions determine the therapeutic utility of BK channel peptide blockers. Blockade of the BK channel induces an increase in neurotransmitter release $^{[94]}$, which suggests that a brain-penetrant blocker of BK channels could increase the signal-to-noise ratio for the remaining synapses, resulting in cognition enhancement in a compromised neuronal system. Other conditions, such as ischemic reperfusion injury, which features abnormal enhancement of BK channel activity indicated by NOS activation ${ }^{[95]}$, epilepsy $^{[21]}$ due to gain-of-function BK channel mutants, or cancer $^{[96]}$ featuring amplifications of the BK channel gene, also represent potential therapeutic uses for BK channel blockers.

\section{Potential clinical development of BK channel non-peptide blockers}

For non-peptide compounds, it is difficult to determine a functional structure because of the large diversity of molecular formulas of these blockers. However, small-molecule blockers such as TEA are also used as pharmacological tools or as potential drugs to increase synaptic transmission and restore cognitive function in some types of neurodegenerative diseases. For example, Phenylindole 57, a BK channel blocker, has been used in a mouse model to reduce depression in a dose-dependent manner ${ }^{[97]}$ and yielded effects similar to those of antidepressant and psychostimulating drugs. Furthermore, Phenylindole 58 enhanced memory when administered by intraperitoneal injection animal models.

Blockers of BK channels may have potential therapeutic uses. However, none of these blockers has been used in clinical treatments due to limitations in synthesis, delivery, metabolism, tissue penetration and reversibility. Therefore, it is important to develop new drugs targeting BK channels that may facilitate the elucidation of the detailed relationships between the structure and function of BK channels. The structures of known blockers can also be used as templates to design new blockers with potential clinical applications.

\section{Acknowledgements}

This work was supported by the National Natural Science Foundation of China (31100847), Youth Innovation Promotion Association CAS (2012323), the Fundamental Research Funds for the Central Universities of China (WK2070000033) to Long-hua ZHANG, and Specialized Research Fund for the Doctoral Program of Higher Education (20113402110058) to Chang-lin TIAN.

\section{References}

1 Wang ZW. Regulation of synaptic transmission by presynaptic CaMKII and BK channels. Mol Neurobiol 2008; 38: 153-66.

2 Wu RS, Marx SO. The BK potassium channel in the vascular smooth muscle and kidney: alpha- and beta-subunits. Kidney Int 2010; 78: 963-74.

3 Wang X, Inukai T, Greer MA, Greer SE. Evidence that $\mathrm{Ca}^{2+}$-activated K ${ }^{+}$ channels participate in the regulation of pituitary prolactin secretion. Brain Res 1994; 662: 83-7.

4 Marty A. Ca-dependent $\mathrm{K}$ channels with large unitary conductance in 
chromaffin cell membranes. Nature 1981; 291: 497-500.

5 Wiecha J, Munz B, Wu Y, Noll T, Tillmanns H, Waldecker B. Blockade of $\mathrm{Ca}^{2+}$-activated $\mathrm{K}^{+}$channels inhibits proliferation of human endothelial cells induced by basic fibroblast growth factor. J Vasc Res 1998; 35: 363-71.

6 Weaver AK, Liu X, Sontheimer H. Role for calcium-activated potassium channels (BK) in growth control of human malignant glioma cells. J Neurosci Res 2004; 78: 224-34.

7 Bloch M, Ousingsawat J, Simon R, Schraml P, Gasser TC, Mihatsch MJ, et al. KCNMA1 gene amplification promotes tumor cell proliferation in human prostate cancer. Oncogene 2007; 26: 2525-34.

8 Salkoff L, Butler A, Ferreira G, Santi C, Wei A. High-conductance potassium channels of the SLO family. Nat Rev Neurosci 2006; 7: 921-31.

9 Contreras GF, Castillo K, Enrique N, Carrasquel-Ursulaez W, Castillo JP, Milesi $\mathrm{V}$, et al. A BK (Slo1) channel journey from molecule to physiology. Channels (Austin) 2013; 7: 442-58.

10 Meera $\mathrm{P}$, Wallner $\mathrm{M}$, Song $\mathrm{M}$, Toro L. Large conductance voltageand calcium-dependent $\mathrm{K}^{+}$channel, a distinct member of voltagedependent ion channels with seven $\mathrm{N}$-terminal transmembrane segments (S0-S6), an extracellular $\mathrm{N}$ terminus, and an intracellular (S9-S10) C terminus. Proc Natl Acad Sci U S A 1997; 94: 14066-71.

11 Wallner M, Meera P, Toro L. Determinant for beta-subunit regulation in high-conductance voltage-activated and $\mathrm{Ca}^{2+}$-sensitive $\mathrm{K}^{+}$channels: an additional transmembrane region at the $\mathrm{N}$ terminus. Proc Natl Acad Sci U S A 1996; 93: 14922-7.

12 Koval OM, Fan Y, Rothberg BS. A role for the SO transmembrane segment in voltage-dependent gating of BK channels. J Gen Physiol 2007; 129: 209-20.

13 Liu HW, Hou PP, Guo XY, Zhao ZW, Hu B, Li X, et al. Structural basis for calcium and magnesium regulation of a large conductance calciumactivated potassium channel with beta1 subunits. J Biol Chem 2014; 289: 16914-23.

14 Dopico AM, Bukiya AN. Lipid regulation of BK channel function. Front Physiol 2014; 5: 312.

15 Shi J, Krishnamoorthy G, Yang Y, Hu L, Chaturvedi N, Harilal D, et al. Mechanism of magnesium activation of calcium-activated potassium channels. Nature 2002; 418: 876-80.

16 Cui J, Yang H, Lee US. Molecular mechanisms of BK channel activation. Cell Mol Life Sci 2009; 66: 852-75.

17 Latorre R, Morera FJ, Zaelzer C. Allosteric interactions and the modular nature of the voltage- and $\mathrm{Ca}^{2+}$-activated (BK) channel. J Physiol 2010; 588: 3141-8.

18 Tomas M, Vazquez E, Fernandez-Fernandez JM, Subirana I, Plata C, Heras M, et al. Genetic variation in the KCNMA1 potassium channel alpha subunit as risk factor for severe essential hypertension and myocardial infarction. J Hypertens 2008; 26: 2147-53.

19 Seibold MA, Wang B, Eng C, Kumar G, Beckman KB, Sen S, et al. An african-specific functional polymorphism in KCNMB1 shows sexspecific association with asthma severity. Hum Mol Genet 2008; 17: 2681-90.

20 McGahon MK, Zhang X, Scholfield CN, Curtis TM, McGeown JG. Selective downregulation of the BKbeta1 subunit in diabetic arteriolar myocytes. Channels (Austin) 2007; 1: 141-3.

21 Du W, Bautista JF, Yang H, Diez-Sampedro A, You SA, Wang L, et al. Calcium-sensitive potassium channelopathy in human epilepsy and paroxysmal movement disorder. Nat Genet 2005; 37: 733-8.

22 Khaitan D, Sankpal UT, Weksler B, Meister EA, Romero IA, Couraud $\mathrm{PO}$, et al. Role of KCNMA1 gene in breast cancer invasion and metastasis to brain. BMC Cancer 2009; 9: 258.

23 Miller C, Moczydlowski E, Latorre R, Phillips M. Charybdotoxin, a protein inhibitor of single $\mathrm{Ca}^{2+}$-activated $\mathrm{K}^{+}$channels from mammalian skeletal muscle. Nature 1985; 313: 316-8.

24 Galvez A, Gimenez-Gallego G, Reuben JP, Roy-Contancin L, Feigenbaum P, Kaczorowski GJ, et al. Purification and characterization of a unique, potent, peptidyl probe for the high conductance calcium-activated potassium channel from venom of the scorpion Buthus tamulus. J Biol Chem 1990; 265: 11083-90.

25 Candia S, Garcia ML, Latorre R. Mode of action of iberiotoxin, a potent blocker of the large conductance $\mathrm{Ca}^{2+}$-activated $\mathrm{K}^{+}$channel. Biophys J 1992; 63: 583-90.

26 Novick J, Leonard RJ, King VF, Schmalhofer W, Kaczorowski GJ. Purification and characterization of two novel peptidyl toxins directed against $\mathrm{K}$ channels from venom of new world scorpions. Biophys $\mathrm{J}$ 1991.

27 Tytgat J, Chandy KG, Garcia ML, Gutman GA, Martin-Eauclaire MF, van der Walt JJ, et al. A unified nomenclature for short-chain peptides isolated from scorpion venoms: alpha-KTx molecular subfamilies. Trends Pharmacol Sci 1999; 20: 444-7.

28 Mackinnon R, Miller C. Mechanism of charybdotoxin block of the high-conductance, $\mathrm{Ca}^{2+}$-activated $\mathrm{K}^{+}$channel. J Gen Physiol 1988; 91: 335-49.

29 Lucchesi K, Ravindran A, Young H, Moczydlowski E. Analysis of the blocking activity of charybdotoxin homologs and iodinated derivatives against $\mathrm{Ca}^{2+}$-activated $\mathrm{K}^{+}$channels. J Membr Biol 1989; 109: 26981.

30 Renisio JG, Lu Z, Blanc E, Jin W, Lewis JH, Bornet O, et al. Solution structure of potassium channel-inhibiting scorpion toxin Lq2. Proteins 1999; 34: 417-26.

31 Miller C. Competition for block of a $\mathrm{Ca}^{2+}$-activated $\mathrm{K}^{+}$channel by charybdotoxin and tetraethylammonium. Neuron 1988; 1 : 1003-6.

32 Bontems F, Roumestand C, Boyot P, Gilquin B, Doljansky Y, Menez A, et al. Three-dimensional structure of natural charybdotoxin in aqueous solution by ${ }^{1} \mathrm{H}-\mathrm{NMR}$. Charybdotoxin possesses a structural motif found in other scorpion toxins. Eur J Biochem 1991; 196: 19-28.

33 MacKinnon R, Latorre R, Miller C. Role of surface electrostatics in the operation of a high-conductance $\mathrm{Ca}^{2+}$-activated $\mathrm{K}^{+}$channel. Biochemistry 1989; 28: 8092-9.

34 Mackinnon R, Miller C. Functional modification of a $\mathrm{Ca}^{2+}$-activated $\mathrm{K}^{+}$ channel by trimethyloxonium. Biochemistry 1989; 28: 8087-92.

35 Park CS, Miller C. Mapping function to structure in a channel-blocking peptide: electrostatic mutants of charybdotoxin. Biochemistry 1992; 31: 7749-55.

36 Stampe P, Kolmakova-Partensky L, Miller C. Intimations of $\mathrm{K}^{+}$channel structure from a complete functional map of the molecular surface of charybdotoxin. Biochemistry 1994; 33: 443-50.

37 Park CS, Miller C. Interaction of charybdotoxin with permeant ions inside the pore of a $\mathrm{K}^{+}$channel. Neuron 1992; 9: 307-13.

38 Hermann A, Erxleben C. Charybdotoxin selectively blocks small Caactivated K channels in Aplysia neurons. J Gen Physiol 1987; 90: 27-47.

39 Attali B, Romey G, Honore E, Schmid-Alliana A, Mattei MG, Lesage F, et al. Cloning, functional expression, and regulation of two $\mathrm{K}^{+}$channels in human T lymphocytes. J Biol Chem 1992; 267: 8650-7.

40 Mackinnon R, Reinhart PH, White MM. Charybdotoxin block of Shaker $\mathrm{K}^{+}$channels suggests that different types of $\mathrm{K}^{+}$channels share common structural features. Neuron 1988; 1: 997-1001.

41 Banerjee A, Lee A, Campbell E, Mackinnon R. Structure of a poreblocking toxin in complex with a eukaryotic voltage-dependent $\mathrm{K}^{+}$ channel. Elife 2013; 2: e00594.

42 Neusch C, Weishaupt JH, Bahr M. Kir channels in the CNS: emerging new roles and implications for neurological diseases. Cell Tissue Res 
2003; 311: 131-8.

43 Johnson BA, Sugg EE. Determination of the three-dimensional structure of iberiotoxin in solution by ${ }^{1} \mathrm{H}$ nuclear magnetic resonance spectroscopy [Comparative Study]. Biochemistry 1992; 31: 8151-9.

44 Ferrat G, Bernard C, Fremont V, Mullmann TJ, Giangiacomo KM, Darbon $\mathrm{H}$. Structural basis for alpha-K toxin specificity for $\mathrm{K}^{+}$channels revealed through the solution ${ }^{1} \mathrm{H}$ NMR structures of two noxiustoxiniberiotoxin chimeras. Biochemistry 2001; 40: 10998-1006.

45 Zheng YM, Park SW, Stokes L, Tang Q, Xiao JH, Wang YX. Distinct activity of BK channel beta1-subunit in cerebral and pulmonary artery smooth muscle cells. Am J Physiol Cell Physiol 2013; 304: C780-9.

46 Zhang J, Halm ST, Halm DR. Role of the BK channel (KCa1.1) during activation of electrogenic $\mathrm{K}^{+}$secretion in guinea pig distal colon. Am J Physiol Gastrointest Liver Physiol 2012; 303: G1322-34.

47 Blanc E, Romi-Lebrun R, Bornet O, Nakajima T, Darbon H. Solution structure of two new toxins from the venom of the Chinese scorpion Buthus martensi Karsch blockers of potassium channels. Biochemistry 1998; 37: 12412-8.

48 Romi-Lebrun R, Lebrun B, Martin-Eauclaire MF, Ishiguro M, Escoubas $\mathrm{P}$, Wu FQ, et al. Purification, characterization, and synthesis of three novel toxins from the Chinese scorpion Buthus martensi, which act on $\mathrm{K}^{+}$channels. Biochemistry 1997; 36: 13473-82.

49 Harvey AL, Vatanpour H, Rowan EG, Pinkasfeld S, Vita C, Menez A, et al. Structure-activity studies on scorpion toxins that block potassium channels. Toxicon 1995; 33: 425-36.

50 Marshall DL, Vatanpour H, Harvey AL, Boyot P, Pinkasfeld S, Doljansky $\mathrm{Y}$, et al. Neuromuscular effects of some potassium channel blocking toxins from the venom of the scorpion Leiurus quinquestriatus hebreus. Toxicon 1994; 32: 1433-43.

51 Garcia-Valdes J, Zamudio FZ, Toro L, Possani LD. Slotoxin, alphaKTx1.11, a new scorpion peptide blocker of MaxiK channels that differentiates between alpha and alpha+beta (beta1 or beta4) complexes. FEBS Lett 2001; 505: 369-73.

52 Crest M, Jacquet G, Gola M, Zerrouk H, Benslimane A, Rochat H, et al. Kaliotoxin, a novel peptidyl inhibitor of neuronal BK-type $\mathrm{Ca}^{2+}$ activated $\mathrm{K}^{+}$channels characterized from Androctonus mauretanicus mauretanicus venom. J Biol Chem 1992; 267: 1640-7.

53 Korukottu J, Schneider R, Vijayan V, Lange A, Pongs O, Becker S, et al. High-resolution 3D structure determination of kaliotoxin by solid-state NMR spectroscopy. PLoS One 2008; 3: e2359.

54 Pentelute BL, Mandal K, Gates ZP, Sawaya MR, Yeates TO, Kent SB. Total chemical synthesis and X-ray structure of kaliotoxin by racemic protein crystallography. Chem Commun (Camb) 2010; 46: 8174-6.

55 Fernandez I, Romi R, Szendeffy S, Martin-Eauclaire MF, Rochat H, Van Rietschoten J, et al. Kaliotoxin (1-37) shows structural differences with related potassium channel blockers. Biochemistry 1994; 33 : 14256-63.

56 Aiyar J, Withka JM, Rizzi JP, Singleton DH, Andrews GC, Lin W, et al. Topology of the pore-region of a $\mathrm{K}^{+}$channel revealed by the NMR-derived structures of scorpion toxins. Neuron 1995; 15: 1169-81.

57 Laraba-Djebari F, Legros C, Crest M, Ceard B, Romi R, Mansuelle P, et al. The kaliotoxin family enlarged. Purification, characterization, and precursor nucleotide sequence of KTX2 from Androctonus australis venom. J Biol Chem 1994; 269: 32835-43.

58 Wang Y, Chen X, Zhang N, Wu G, Wu H. The solution structure of BmTx3B, a member of the scorpion toxin subfamily alpha-KTx 16. Proteins 2005; 58: 489-97.

59 Tao J, Zhou ZL, Wu B, Shi J, Chen XM, Ji YH. Recombinant expression and functional characterization of martentoxin: a selective inhibitor for BK channel (alpha + beta4). Toxins (Basel) 2014; 6: 1419-33.

60 Shi J, He HQ, Zhao R, Duan YH, Chen J, Chen Y, et al. Inhibition of martentoxin on neuronal BK channel subtype (alpha+beta4): implications for a novel interaction model. Biophys J 2008; 94: 3706-13.

61 Tao J, Shi J, Yan L, Chen Y, Duan YH, Ye P, et al. Enhancement effects of martentoxin on glioma BK channel and BK channel (alpha+beta1) subtypes. PLoS One 2011; 6: e15896.

62 Cai Z, Xu C, Xu Y, Lu W, Chi CW, Shi Y, et al. Solution structure of BmBKTx1, a new BKCa1 channel blocker from the Chinese scorpion Buthus martensi Karsch. Biochemistry 2004; 43: 3764-71.

63 Xu CQ, Brone B, Wicher D, Bozkurt O, Lu WY, Huys I, et al. BmBKTx1, a novel $\mathrm{Ca}^{2+}$-activated $\mathrm{K}^{+}$channel blocker purified from the Asian scorpion Buthus martensi Karsch. J Biol Chem 2004; 279: 34562-9.

64 Holaday SK Jr, Martin BM, Fletcher PL Jr, Krishna NR. NMR solution structure of butantoxin. Arch Biochem Biophys 2000; 379: 18-27.

65 M'Barek S, Chagot B, Andreotti N, Visan V, Mansuelle P, Grissmer S, et al. Increasing the molecular contacts between maurotoxin and Kv1.2 channel augments ligand affinity. Proteins 2005; 60: 401-11.

66 Novello JC, Arantes EC, Varanda WA, Oliveira B, Giglio JR, Marangoni S. TsTX-IV, a short chain four-disulfide-bridged neurotoxin from Tityus serrulatus venom which acts on $\mathrm{Ca}^{2+}$-activated $\mathrm{K}^{+}$channels. Toxicon 1999; 37: 651-60.

67 Yao J, Chen X, Li H, Zhou Y, Yao L, Wu G, et al. BmP09, a "long chain" scorpion peptide blocker of BK channels. J Biol Chem 2005; 280 : 14819-28.

68 Wang J, Shen B, Guo M, Lou X, Duan Y, Cheng XP, et al. Blocking effect and crystal structure of natrin toxin, a cysteine-rich secretory protein from Naja atra venom that targets the BKCa channel. Biochemistry 2005; 44: 10145-52.

69 Tu X, Wang J, Guo M, Zheng D, Teng M, Niu L, et al. Purification, partial characterization, crystallization and preliminary $X$-ray diffraction of two cysteine-rich secretory proteins from Naja atra and Trimeresurus stejnegeri venoms. Acta Crystallogr D Biol Crystallogr 2004; 60: 1108-11.

70 Garcia ML, Hanner M, Knaus HG, Slaughter R, Kaczorowski GJ. Scorpion toxins as tools for studying potassium channels. Methods Enzymol 1999; 294: 624-39.

71 Park CS, Hausdorff SF, Miller C. Design, synthesis, and functional expression of a gene for charybdotoxin, a peptide blocker of $\mathrm{K}^{+}$ channels. Proc Natl Acad Sci U S A 1991; 88: 2046-50.

72 Bende NS, Dziemborowicz S, Mobli M, Herzig V, Gilchrist J, Wagner J, et al. A distinct sodium channel voltage-sensor locus determines insect selectivity of the spider toxin Dc1a. Nat Commun 2014; 5: 4350.

73 Schilling S, Wasternack C, Demuth HU. Glutaminyl cyclases from animals and plants: a case of functionally convergent protein evolution. Biol Chem 2008; 389: 983-91.

74 Kaczorowski GJ, Knaus HG, Leonard RJ, McManus OB, Garcia ML. High-conductance calcium-activated potassium channels; structure, pharmacology, and function. J Bioenerg Biomembr 1996; 28: 25567.

75 Nardi A, Calderone V, Chericoni S, Morelli I. Natural modulators of large-conductance calcium-activated potassium channels. Planta Med 2003; 69: 885-92.

76 John E, Starrett J, Steven I, Dworetzky, Valentin K, Gribkoff. Modulators of large-conductance calcium-activated potassium (BK) channels as potential therapeutic targets. Current Pharmaceeutical Design 1996; 2: 413-28.

77 Knaus HG, McManus OB, Lee SH, Schmalhofer WA, Garcia-Calvo M, Helms LM, et al. Tremorgenic indole alkaloids potently inhibit smooth muscle high-conductance calcium-activated potassium channels. Biochemistry 1994; 33: 5819-28.

78 Cole RJ, Cox RH. Handbook of Toxic Fungal Metabolites. 1981: 355509. 
79 Norris PJ, Smith CC, De Belleroche J, Bradford HF, Mantle PG, Thomas $\mathrm{AJ}$, et al. Actions of tremorgenic fungal toxins on neurotransmitter release. J Neurochem 1980; 34: 33-42.

80 Villarroel A, Alvarez O, Oberhauser A, Latorre R. Probing a $\mathrm{Ca}^{2+}$ activated $\mathrm{K}^{+}$channel with quaternary ammonium ions. Pflugers Arch 1988; 413: 118-26.

81 Harper AA, Catacuzzeno L, Trequattrini C, Petris A, Franciolini F. Verapamil block of large-conductance Ca-activated $\mathrm{K}$ channels in rat aortic myocytes. J Membr Biol 2001; 179: 103-11.

82 Wickenden A. $\mathrm{K}^{+}$channels as therapeutic drug targets. Pharmacol Ther 2002; 94: 157-82.

83 Marcie G. Berger NJR. Voltage and calcium-gated potassium channels: Functional expression and therapeutic potential in the vasculature. Perspectives in Drug Discovery and Design 1999; 15-16: 313-32.

84 Wang G, Lemos JR. Tetrandrine blocks a slow, large-conductance, $\mathrm{Ca}^{2+}$-activated potassium channel besides inhibiting a non-inactivating $\mathrm{Ca}^{2+}$ current in isolated nerve terminals of the rat neurohypophysis. Pflugers Arch 1992; 421: 558-65.

85 Wu SN, Li HF, Lo YC. Characterization of tetrandrine-induced inhibition of large-conductance calcium-activated potassium channels in a human endothelial cell line (HUV-EC-C). J Pharmacol Exp Ther 2000; 292: 188-95.

86 Wu SN, Li HF, Jan CR, Shen AY. Inhibition of $\mathrm{Ca}^{2+}$-activated $\mathrm{K}^{+}$current by clotrimazole in rat anterior pituitary GH3 cells. Neuropharmacology 1999; 38: 979-89.

87 Chiang HT, Wu SN. Inhibition of large-conductance calcium-activated potassium channel by 2-methoxyestradiol in cultured vascular endothelial (HUV-EC-C) cells. J Membr Biol 2001; 182: 203-12.

88 Liu YC, Lo YC, Huang CW, Wu SN. Inhibitory action of ICl-182,780, an estrogen receptor antagonist, on $\mathrm{BK}_{\mathrm{ca}}$ channel activity in cultured endothelial cells of human coronary artery. Biochem Pharmacol 2003;
66: 2053-63.

89 Zhou Y, Lingle CJ. Paxilline inhibits BK channels by an almost exclusively closed-channel block mechanism. J Gen Physiol 2014; 144: 415-40.

90 Anderson CS, MacKinnon R, Smith C, Miller C. Charybdotoxin block of single $\mathrm{Ca}^{2+}$-activated $\mathrm{K}^{+}$channels. Effects of channel gating, voltage, and ionic strength. J Gen Physiol 1988; 91: 317-33.

91 Giangiacomo KM, Garcia ML, McManus OB. Mechanism of iberiotoxin block of the large-conductance calcium-activated potassium channel from bovine aortic smooth muscle. Biochemistry 1992; 31: 6719-27.

92 Schroeder N, Mullmann TJ, Schmalhofer WA, Gao YD, Garcia ML, Giangiacomo KM. Glycine 30 in iberiotoxin is a critical determinant of its specificity for maxi-K versus $K_{v}$ channels. FEBS Lett 2002; 527 : 298-302.

93 Gao YD, Garcia ML. Interaction of agitoxin2, charybdotoxin, and iberiotoxin with potassium channels: selectivity between voltage-gated and Maxi-K channels. Proteins 2003; 52: 146-54.

$94 \mathrm{Hu} \mathrm{H}$, Shao LR, Chavoshy S, Gu N, Trieb M, Behrens R, et al. Presynaptic $\mathrm{Ca}^{2+}$-activated $\mathrm{K}^{+}$channels in glutamatergic hippocampal terminals and their role in spike repolarization and regulation of transmitter release. J Neurosci 2001; 21: 9585-97.

95 Tricarico D, Capriulo R, Camerino DC. Involvement of $\mathrm{K}_{\mathrm{Ca} 2+}$ channels in the local abnormalities and hyperkalemia following the ischemiareperfusion injury of rat skeletal muscle. Neuromuscul Disord 2002; 12: $258-65$

96 Ge L, Hoa NT, Wilson Z, Arismendi-Morillo G, Kong XT, Tajhya RB, et al. Big Potassium (BK) ion channels in biology, disease and possible targets for cancer immunotherapy. Int Immunopharmacol 2014; 22 : 427-43.

97 Olesen SP, Munch E, Moldt P, Drejer J. Selective activation of $\mathrm{Ca}^{2+}$ dependent $\mathrm{K}^{+}$channels by novel benzimidazolone. Eur J Pharmacol 1994; 251: 53-9. 